\title{
Modeling the current density generated by transcutaneous spinal direct current stimulation (tsDCS)
}

\author{
Marta Parazzini a,*, Serena Fiocchi a , Ilaria Liorni ${ }^{\mathrm{a}, \mathrm{b}}$, Elena Rossi ${ }^{\mathrm{b}, \mathrm{c}}$, Filippo Cogiamanian ${ }^{\mathrm{c}, \mathrm{d}}$, \\ Maurizio Vergari ${ }^{c, d}$, Alberto Priori ${ }^{c, d}$, Paolo Ravazzani ${ }^{\mathrm{a}}$ \\ ${ }^{a}$ CNR Consiglio Nazionale delle Ricerche, Istituto di Elettronica e di Ingegneria dell'Informazione e delle Telecomunicazioni IEIIT, 20133 Milano, Italy \\ b Dipartimento di Elettronica, Informazione e Bioingegneria, Politecnico di Milano, 20133 Milano, Italy \\ ' Dipartimento di Fisiopatologia Medico-Chirurgica e dei Trapianti, Università degli Studi di Milano, 20122 Milano, Italy \\ ${ }^{\mathrm{d}}$ Centro Clinico per la Neurostimolazione, le Neurotecnologie ed i Disordini del Movimento, Fondazione IRCCS Ca' Granda Ospedale Maggiore Policlinico, 20122 Milano, Italy
}

Accepted 24 February 2014

Available online 3 April 2014

\section{Introduction}

The first evidence that the application of direct current stimulation over the spinal cord (transcutaneous spinal DC stimulation, tsDCS) can modulate conduction along the spinal somatosensory pathways in humans was provided by the first study of

E-mail address: marta.parazzini@ieiit.cnr.it (M. Parazzini). 
Cogiamanian et al. (2008). They showed that thoracic anodal tsDCS (2.5 $\mathrm{mA}$ for $15 \mathrm{~min}$ ) selectively reduced the cervico-medullary component of the posterior tibial nerve somatosensory-evoked potential for at least $20 \mathrm{~min}$ after the end of the stimulation. Afterwards, further studies in healthy subjects supported the possibility that tsDCS can alter spinal cord functions (for a review see Cogiamanian et al., 2012). Briefly, it has been shown that tsDCS can (i) modulate the processing of nociceptive spinal pathways as it reduces both foot-laser-evoked potentials (Truini et al., 2011) and the RIII nociceptive component of the flexion reflex in the lower limb (Cogiamanian et al., 2011); and (ii) alter spinal motor circuits since it induces both a lasting decrease in H-reflex post-activation depression (Winkler et al., 2010) and a progressive left-ward shift of the stimulus-response curve of the soleus $\mathrm{H}$ reflex (Lamy et al., 2012). Spinal DC stimulation applied in animals has suggested that (i) tsDCS could affect intrinsic properties of the motoneurons (Ahmed, 2011); (ii) that DC-induced effects on spinal neuronal circuits could be mediated by changes in the glutamatergic

Table 1

Characteristics of the anatomical models.

\begin{tabular}{llllll}
\hline Name & Sex & Age $(\mathrm{y})$ & Height $(\mathrm{m})$ & Weight $(\mathrm{kg})$ & No. of tissues \\
\hline Ella & Female & 26 & 1.63 & 59 & 76 \\
Louis & Male & 14 & 1.69 & 50.4 & 77 \\
Billie & Female & 11 & 1.47 & 35 & 75 \\
\hline
\end{tabular}

Table 2

Tissues conductivity.

\begin{tabular}{|c|c|}
\hline Tissue & $\begin{array}{l}\text { Conductivity } \\
(\mathrm{S} / \mathrm{m})\end{array}$ \\
\hline $\begin{array}{l}\text { Adrenal gland, epididymis, esophagus, hypophysis, pancreas, } \\
\text { pineal body, small intestine, stomach, thymus, thyroid } \\
\text { gland }\end{array}$ & 0.51113 \\
\hline Air internal, bronchi lumen, pharynx & 0 \\
\hline Artery, blood vessel, heart lumen, penis, vein & 0.6 \\
\hline Bladder & 0.202783 \\
\hline Bone, mandible, marrow red, patella, skull, teeth, vertebrae & 0.020 \\
\hline Breast & 0.26167 \\
\hline Bronchi & 0.250552 \\
\hline Cartilage, ear cartilage, intervertebral disks, larynx, trachea & 0.16113 \\
\hline Cerebellum & 0.047512 \\
\hline CSF & 1.59 \\
\hline Connective tissue & 0.16446 \\
\hline Cornea, prostate, testis & 0.4113 \\
\hline Diaphragm & 0.201967 \\
\hline Eye sclera & 0.501392 \\
\hline Eye-vitreous humor & 1.5 \\
\hline Eye-lens & 0.3113 \\
\hline Fat, subcutaneous adipose tissue (SAT) & 0.078 \\
\hline Gallbladder & 1.27 \\
\hline Grey matter, hippocampus, hypothalamus, thalamus & 0.027512 \\
\hline Heart lumen & 0.6 \\
\hline Heart muscle & 0.48 \\
\hline Hypophisys, pineal body & 0.511 \\
\hline Kidney cortex, kidney medulla & 0.0544105 \\
\hline Large intestine, Large intestine lumen & 0.0122052 \\
\hline Liver & 0.091 \\
\hline Lung & 0.076 \\
\hline Medulla oblongata, Midbrain, pons & 0.0276 \\
\hline Meniscus, trachea & 0.16113 \\
\hline Mucosa & 0.0004 \\
\hline Muscle & 0.16 \\
\hline Nerve, spinal cord & 0.0171267 \\
\hline Ovary & 0.31113 \\
\hline Skin, ear-skin & 0.1 \\
\hline Spleen & 0.0395962 \\
\hline Tendon ligament & 0.250922 \\
\hline Tongue & 0.26113 \\
\hline Ureter-urethra & 0.25055 \\
\hline Uterus & 0.201296 \\
\hline Vagina & 0.0122052 \\
\hline White matter, commissura anterior, commissura posterior & 0.027656 \\
\hline
\end{tabular}

neurotransmission (Ahmed and Wieraszko, 2012); (iii) that cathodal tsDCS could amplify cortically evoked movements through spinal mechanisms (Ahmed, 2013a); (iv) that cathodal tsDCS could modulate associative plasticity and combined cortical and peripheral associative stimulation, accompanied by cathodal tsDCS, can improve skilled locomotor recovery after spinal cord injury (SCI) in mice (Ahmed, 2013b).

Recently, Hubli et al. (2013) applied tsDCS to patients with complete motor $\mathrm{SCI}$, showing that spinal reflex amplitude is enhanced after a single session of anodal tsDCS in SCI patients but unchanged following cathodal, sham tsDCS or assisted locomotion, whereas both anodal tsDCS and assisted locomotion reduced spinal reflex threshold. This suggests the possibility that tsDCS could be an innovative noninvasive neuromodulatory tool to prevent neuronal dysfunction developing after SCl. However, this possibility needs further investigations.

Recently, the integration between clinical studies and computational forward models of brain current flow during transcranial direct current stimulation (tDCS) has shown that DC stimulationinduced aftereffects depend on the electrodes montages, on the current density reaching the target tissue, on the neural circuits involved and on the biophysical properties of neuronal membranes (Brunoni et al., 2012; Lamy, 2013). This has led to a better optimization of clinical electrotherapy.

Therefore, following the approach that turned out extremely efficient in tDCS, we here propose the first study aiming to estimate the current density distributions in the spinal cord by applying electromagnetic computation techniques to human models and varying the electrode montages.

This study is important for three main reasons: (i) it is the first study that describes the current flow along the spine due to tsDCS, therefore it will form the basis for future models exploring different electrode montages before using them on patients; (ii) it can be useful in guiding the clinical applications of DC spinal cord stimulation and (iii) these data it could be useful also in interpreting

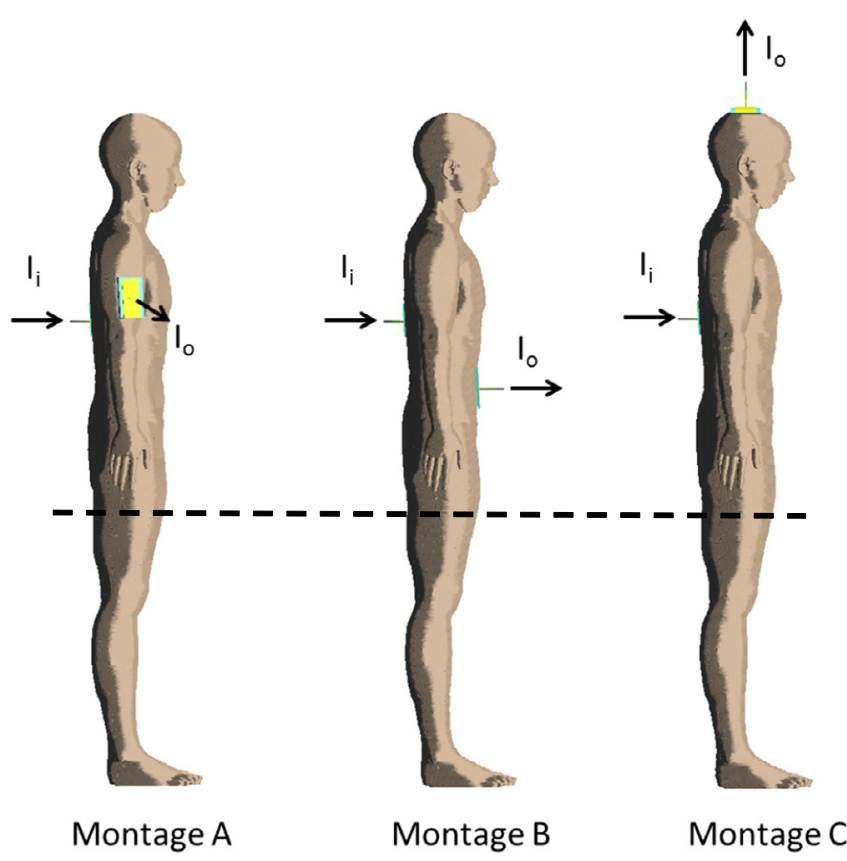

Fig. 1. The three electrode montages shown on "Ella" model. The yellow rectangular pad is the conductor, while the light blue rectangular pad is the sponge. The wires attached to the electrode are used to estimate the injected and extracted current. The dashed line shows where the models are truncated for the simulations. Legend: Ii: input current; Io: output current. (For interpretation of the references to color in this figure legend, the reader is referred to the web version of this article.) 
some physiological data already available for the specific montages here modeled.

\section{Materials and methods}

Simulations were conducted using the simulation platform SEMCAD X (by SPEAG, Schmid \& Partner Engineering, AG, Zurich, Switzerland, www.speag.com), solving the Laplace equation to determine the electric potential $(\varphi)$ distribution inside the human tissues

$\nabla \cdot(\sigma \nabla \varphi)=0$ where $\sigma$ is the electrical conductivity of the human tissues. The $\mathbf{E}$ and $\mathbf{J}$ field distributions were obtained by means of the following relations:

$\mathbf{E}=-\nabla \varphi$

$\mathbf{J}=\sigma \mathbf{E}$

Three human realistic models of the "Virtual Population" (Christ et al., 2010) were used. These models have been developed from high-resolution magnetic resonance images of healthy volunteers ("Ella", "Louis", "Billie") whose characteristics are reported in Table 1. All models are based on the computer-aided design repre-

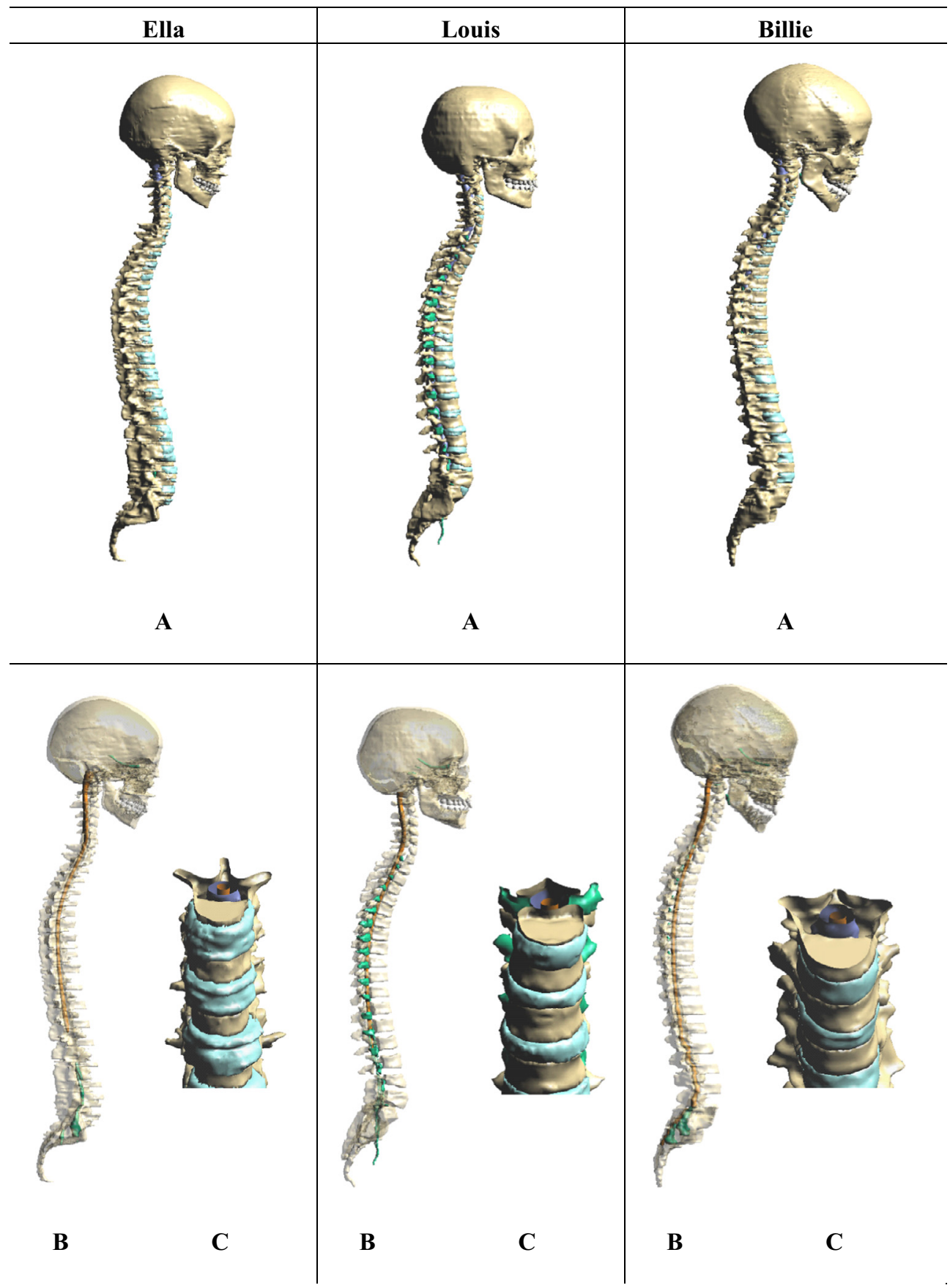

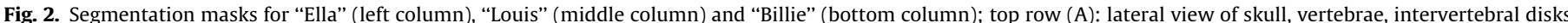

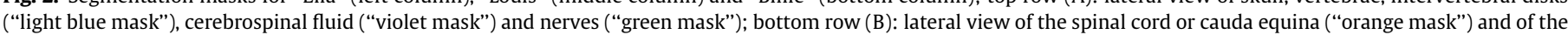

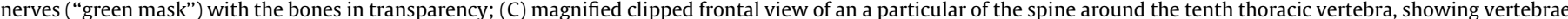

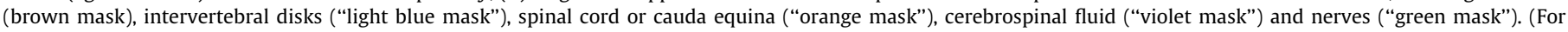
interpretation of the references to color in this figure legend, the reader is referred to the web version of this article.) 
sentation of the organ surfaces. A detailed description of the construction of the models is given in Christ et al. (2010). The dielectric properties of each tissue were assigned on the basis of the data at low frequency (Gabriel et al., 1996, 2009) with the exception of the skin. This latter was modeled as a weighted average of the electrical conductivities of the skin and of the subcutaneous adipose tissue, which is the tissue just below the skin, following an approach already use in literature (Parazzini et al., 2011, 2012) to take into account the higher conductivity of deeper granular tissue (Gabriel, 1997; Dimbylow, 2005). Since the models contain more tissues than what are available in the literature database on electrical conductivities (Gabriel et al., 1996, 2009; Dimbylow, 2005) we assigned the dielectric properties according to the "recommended tissues' correspondence" of the Virtual Population itself (Christ et al., 2010). Table 2 summarizes the conductivities assigned to the different tissues.
For each model, three electrode montages were modeled in which the active electrode was always over the spinal process of the tenth thoracic vertebra and the reference was placed: (i) above the right arm (Montage A); (ii) over the umbilicus (Montage B); (iii) over $\mathrm{Cz}$ (Montage $\mathrm{C}$ ). While Montage $\mathrm{A}$ has been already used in different studies (see the review of Cogiamanian et al., 2012), the other two are still pilot montages and have been used only in one preliminary study on 6 subjects (Vergari et al., 2012). The electrodes were modeled as rectangular pads conductors $(\sigma=5.9 \times$ $\left.10^{7} \mathrm{~S} / \mathrm{m}\right)$ of $5 \times 7.5 \mathrm{~cm}^{2}$ or $5 \times 9.5 \mathrm{~cm}^{2}$ placed above a rectangular sponge $(\sigma=1.4 \mathrm{~S} / \mathrm{m})$ of $7 \times 8 \mathrm{~cm}^{2}$ or $7 \times 10 \mathrm{~cm}^{2}$, for the active and reference electrode, respectively. Fig. 1 shows a lateral view of the three electrodes positioning on "Ella" here used as example. The potential difference between the electrodes was adjusted to inject a total current of $3 \mathrm{~mA}$. This value corresponds to the maximum current intensity tested in our clinical setting up till now

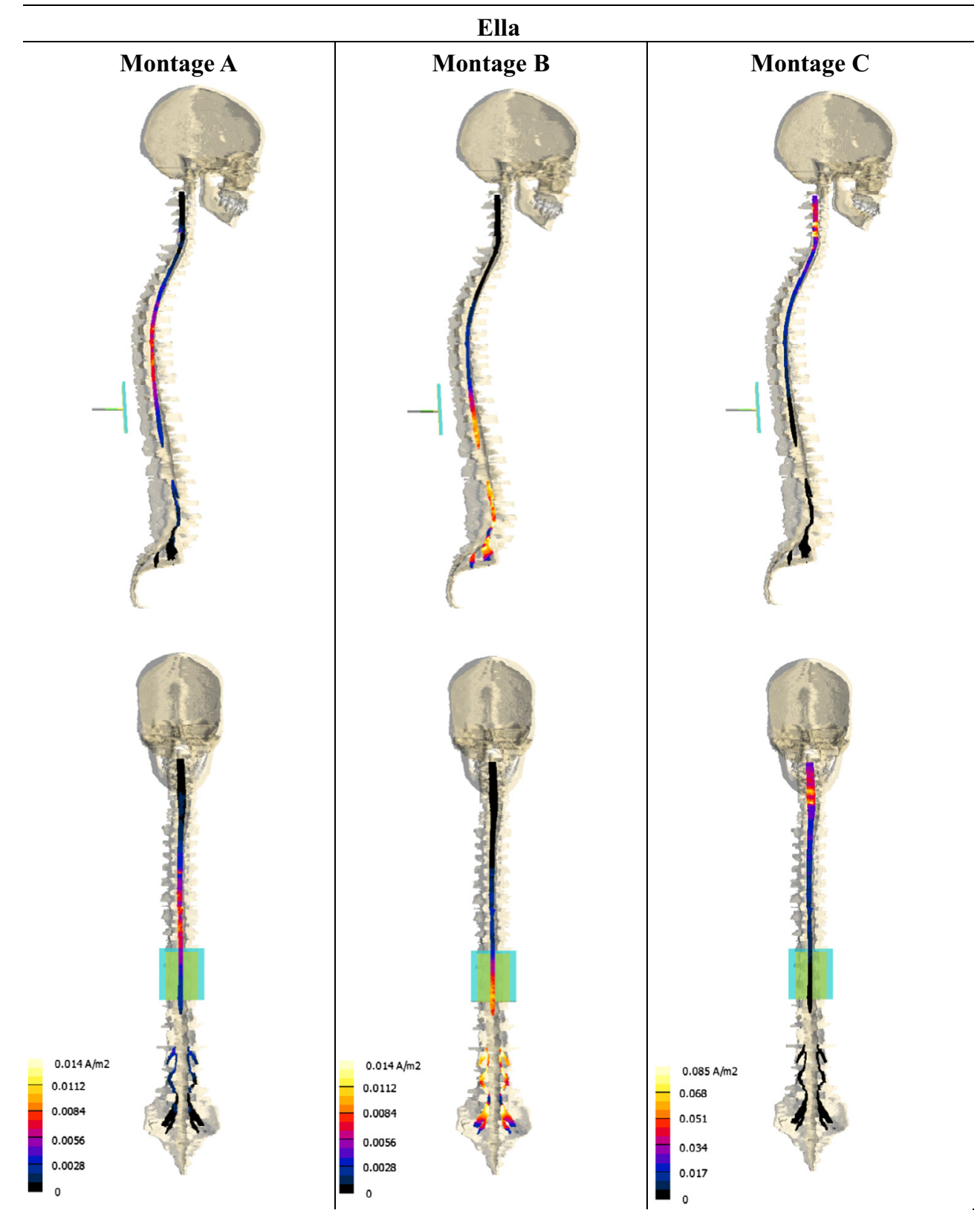

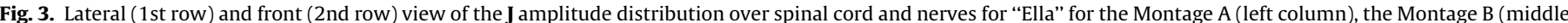

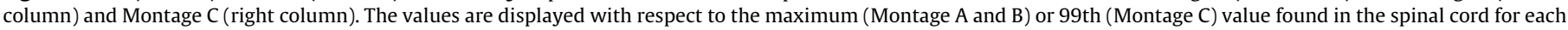
electrode montage. 
(personal communication). In any case, the effect of changing the injected current intensities is a linearly correlated change of the current density distribution. The current value was computed by integrating the current density on the wires surfaces attached to the electrodes (see Fig. 1). For each simulation, the human model and the electrodes were inserted in a surrounding bounding box filled with air and all the models were trunked at the thigh level (see Fig. 1). The boundaries of the bounding box were treated as insulated, i.e., the normal component of the current density was set equal to zero $(J \cdot n=0)$. Continuity of the tangential component of $\mathbf{E}$ was applied at each tissue-to-tissue boundary $\left(E_{\mathrm{t} 1}=E_{\mathrm{t} 2}\right.$; which is equivalent to $\left.J_{\mathrm{t} 1} / \sigma_{1}=J_{\mathrm{t} 2} / \sigma_{2}\right)$. At the interface between the skin and the air the current density was set to be parallel to the face (Parazzini et al., 2011, 2012).

The amplitude distributions of $\mathbf{J}$ were analyzed in the spinal region, particularly on the spinal cord, the cauda equina, the nerves roots and the muscles of back along the spine. In their most recent guidelines for limiting exposure to time-varying electric and magnetic fields (ICNIRP, 2010), the International Commission on NonIonizing Radiation Protection (ICNIRP) recommends determining the induced electric field as a vector average of the electric field in a small contiguous tissue volume of $2 \times 2 \times 2 \mathrm{~mm}^{3}$, as a practical compromise satisfying both requirements for a sound biological basis and computational constraints (ICNIRP, 2010). Therefore, in the following we will use this definition for the $\mathbf{E}$ field distribution. The $\mathbf{J}$ field distribution will be consequently obtained by Eq. (3) above.

The anatomical characteristics of the spine of each model are shown in Fig. 2 where all the segmented spinal tissues masks (i.e., vertebrae, intervertebral disks, cerebrospinal fluid, spinal cord, cauda equina and nerves) are depicted for each model. It is to note that all the models are affected by some limitations of the spinal masks due to segmentation artefacts/problems. For example, the

Louis

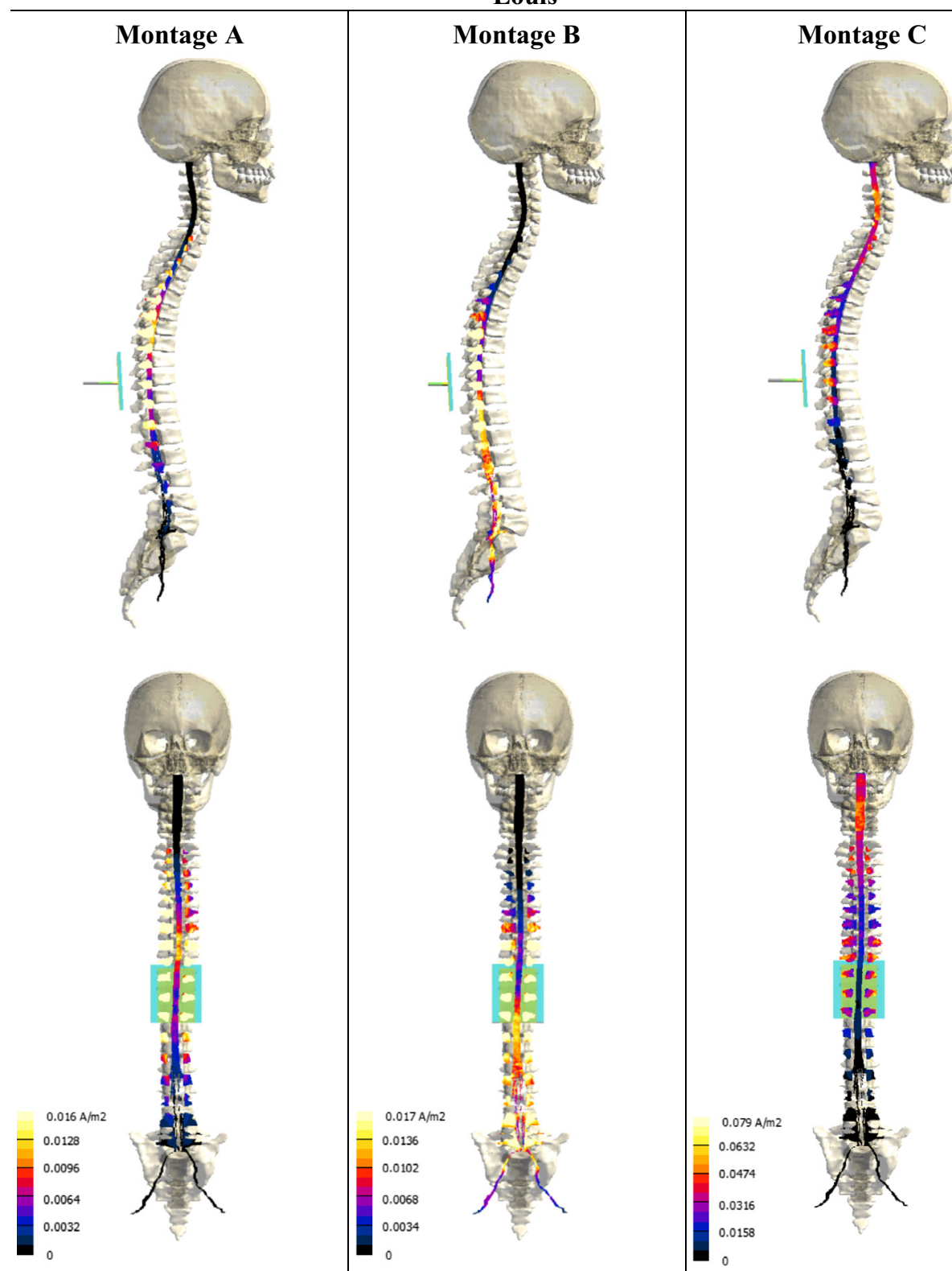

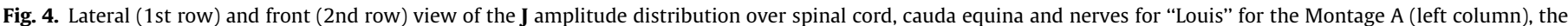

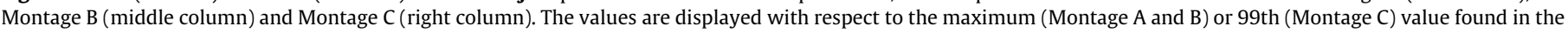
spinal cord for each electrode montage. 
cauda equina of "Ella" is not segmented, for "Louis" it is segmented up to the lumbar level whereas for "Billie" is almost totally segmented; the nerves roots are well defined along all the spine only in "Louis" whereas, on the contrary, the beginning of the sciatic nerve is more evident in "Ella" and "Billie". Only "Louis" shows also part of the sacral plexus. Therefore, in the interpretation of the results, all this differences will be taken into account.

In the following, for clarity, the different levels along the spine will be identified using the name of the corresponding vertebrae (i.e. cervical, thoracic, lumbar, sacral \& coccygeal).

\section{Results}

Figs. 3-5 show the front and lateral views of the amplitude distribution of $\mathbf{J}$ on the surfaces of the spinal cord, cauda equina and nerves for the three electrode montages (Montage A, B and C) and for each human model, whereas Fig. 6 shows the amplitude distribution of $\mathbf{J}$ over a sagittal, coronal and transversal section across the spinal cord only for "Louis" for all the electrode montages. In all the panels, the distributions are shown respect to the maximum or the 99th percentile found in the spinal cord or the cauda equina in each electrode montage. The 99th percentile was chosen instead of the maximum only in the case in which the maximum value was clearly identifiable as a spurious point and mainly due to staircase error. This is clearly stated in the figures' legends. For the sake of brevity, in the following we will use always the word maximum. A qualitative comparison of these panels indicates that varying the cathode position induces different $\mathbf{J}$-field amplitude distributions along the spinal cord, the cauda equina and the nerve roots. Despite inter-individual differences, the pattern of these distributions across the different models and spinal

Billie

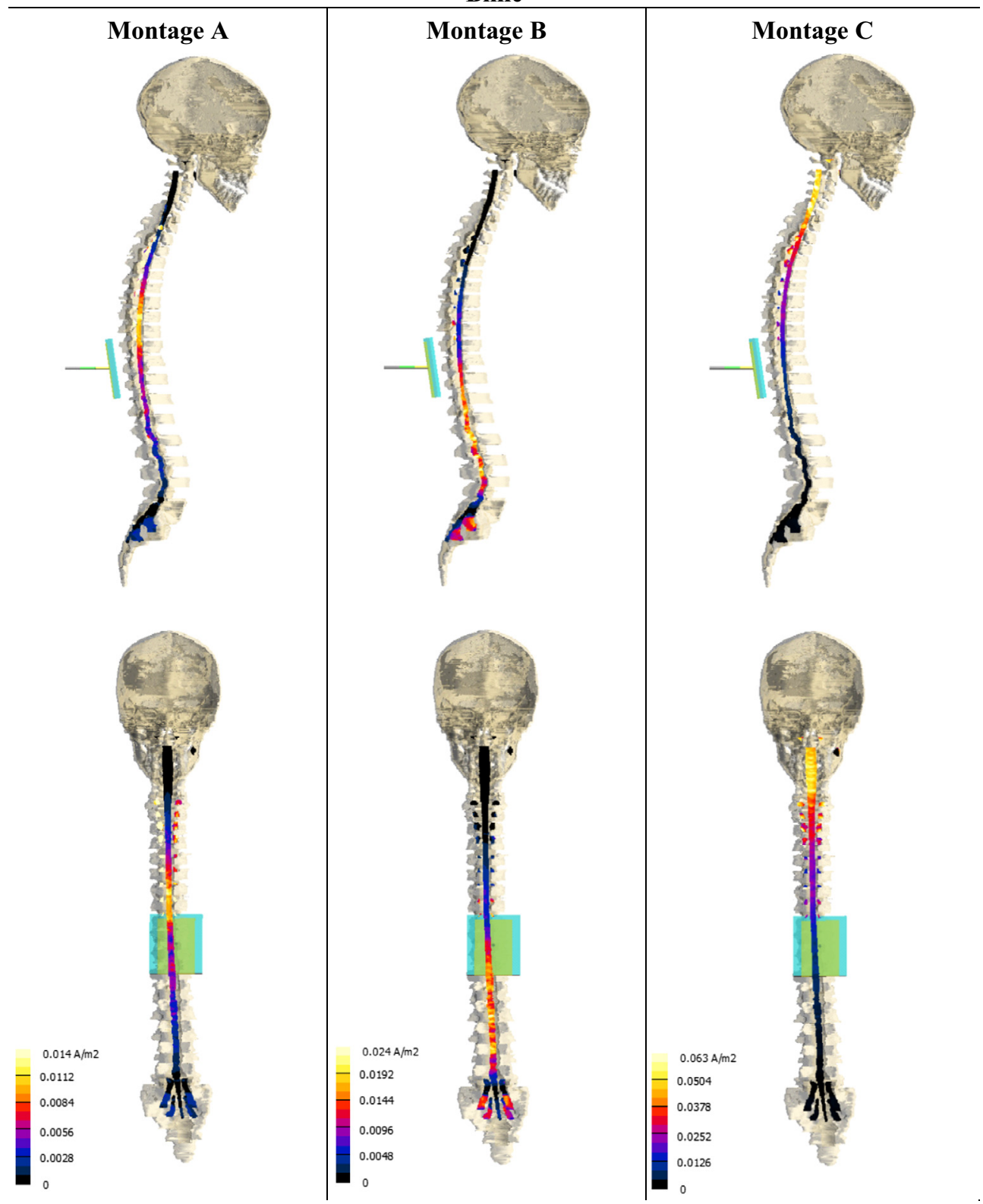

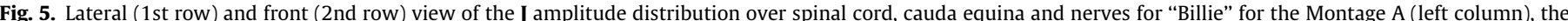

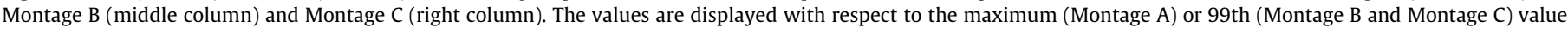
found in the spinal cord for each electrode montage. 


\section{Louis}

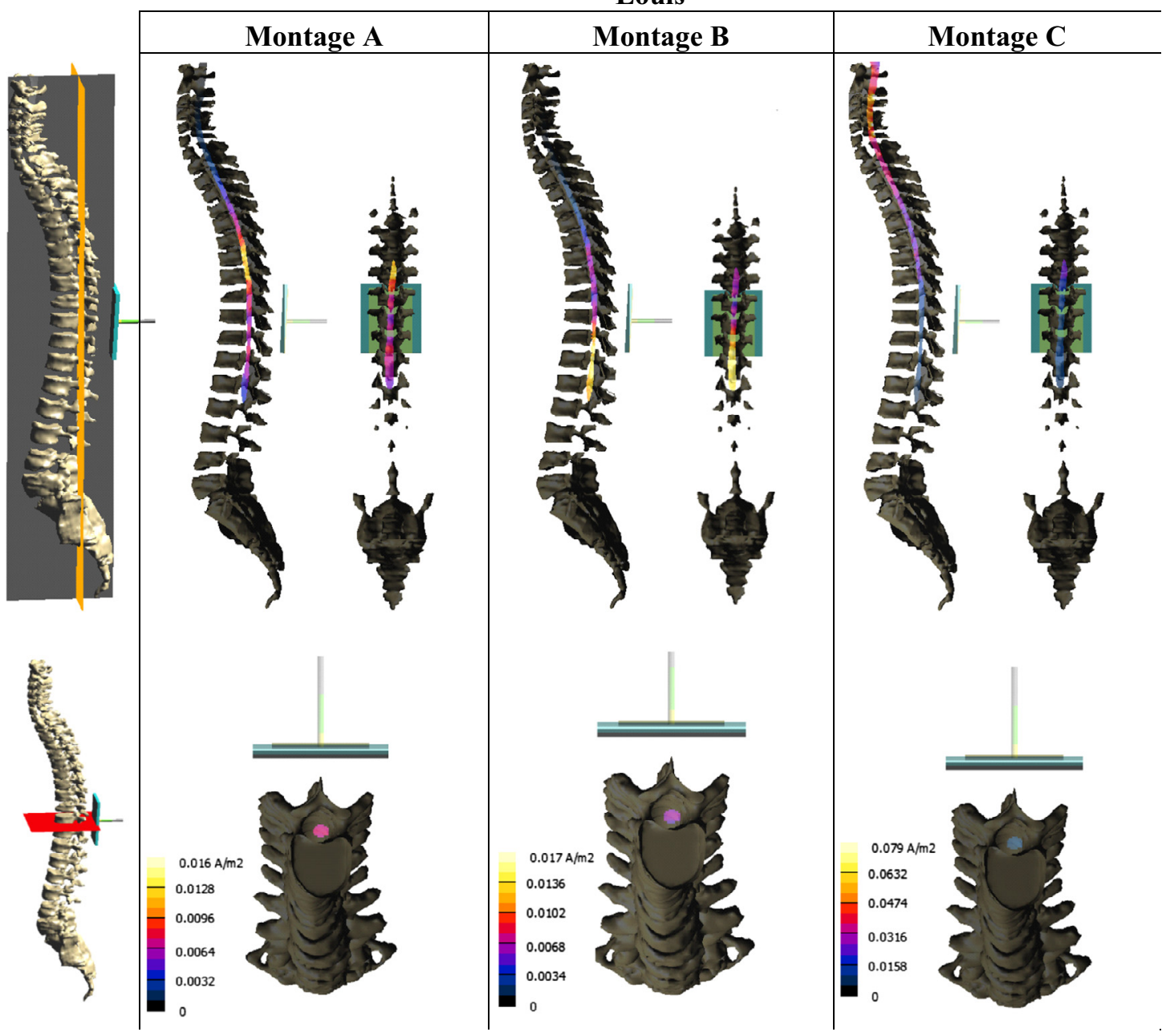

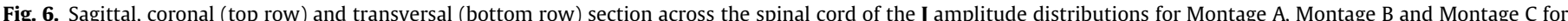

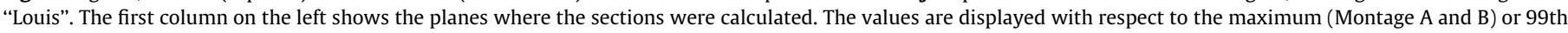
(Montage $\mathrm{C}$ ) value found in the spinal cord for each electrode montage.

Table 3

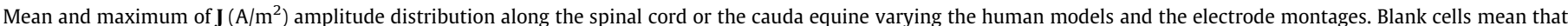
the spinal cord or the cauda equine at that level along the spine is not segmented.

\begin{tabular}{|c|c|c|c|c|c|c|}
\hline & \multicolumn{2}{|c|}{ Montage A } & \multicolumn{2}{|c|}{ Montage B } & \multicolumn{2}{|c|}{ Montage C } \\
\hline & Mean & Max & Mean & Max & Mean & Max \\
\hline \multicolumn{7}{|l|}{ Ella } \\
\hline Cervical & $8.2 \mathrm{E}-04$ & $6.4 \mathrm{E}-03$ & $5.7 \mathrm{E}-05$ & $3.2 \mathrm{E}-04$ & $3.4 \mathrm{E}-02$ & $8.5 \mathrm{E}-02$ \\
\hline Thoracic & $4.6 \mathrm{E}-03$ & $1.4 \mathrm{E}-02$ & $4.1 \mathrm{E}-03$ & $1.4 \mathrm{E}-02$ & $9.4 \mathrm{E}-03$ & $2.8 \mathrm{E}-02$ \\
\hline \multicolumn{7}{|l|}{ Lumbar } \\
\hline \multicolumn{7}{|l|}{ Sacral \& coccygeal } \\
\hline \multicolumn{7}{|l|}{ Louis } \\
\hline Cervical & $3.6 \mathrm{E}-04$ & $2.4 \mathrm{E}-03$ & $4.7 \mathrm{E}-05$ & $4.1 \mathrm{E}-04$ & $3.4 \mathrm{E}-02$ & $7.9 \mathrm{E}-02$ \\
\hline Thoracic & $5.4 \mathrm{E}-03$ & $1.6 \mathrm{E}-02$ & $4.9 \mathrm{E}-03$ & $1.6 \mathrm{E}-02$ & $1.6 \mathrm{E}-02$ & $3.3 \mathrm{E}-02$ \\
\hline Lumbar & $3.9 \mathrm{E}-03$ & $6.1 \mathrm{E}-03$ & $1.2 \mathrm{E}-02$ & $1.7 \mathrm{E}-02$ & $3.8 \mathrm{E}-03$ & $6.0 \mathrm{E}-03$ \\
\hline \multicolumn{7}{|l|}{ Sacral \& coccygeal } \\
\hline \multicolumn{7}{|l|}{ Billie } \\
\hline Cervical & $6.5 \mathrm{E}-04$ & $3.4 \mathrm{E}-03$ & $1.5 \mathrm{E}-04$ & $5.2 \mathrm{E}-04$ & $4.0 \mathrm{E}-02$ & $6.3 \mathrm{E}-02$ \\
\hline Thoracic & $6.3 \mathrm{E}-03$ & $1.4 \mathrm{E}-02$ & $5.8 \mathrm{E}-03$ & $1.9 \mathrm{E}-02$ & $1.4 \mathrm{E}-02$ & $3.2 \mathrm{E}-02$ \\
\hline Lumbar & $2.3 \mathrm{E}-03$ & $1.1 \mathrm{E}-02$ & $1.0 \mathrm{E}-02$ & $2.4 \mathrm{E}-02$ & $1.6 \mathrm{E}-03$ & $8.5 \mathrm{E}-03$ \\
\hline Sacral \& coccygeal & $9.2 \mathrm{E}-04$ & $1.7 \mathrm{E}-03$ & $2.3 \mathrm{E}-03$ & $4.3 \mathrm{E}-03$ & $2.5 \mathrm{E}-04$ & $4.6 \mathrm{E}-04$ \\
\hline
\end{tabular}

structure is qualitatively comparable. Along the vertebral column, Montage A tends to have higher amplitude values in the spinal cord at thoracic level, whereas the Montage $\mathrm{B}$ and $\mathrm{C}$ generate higher amplitude values in the cauda equina at lumbar level and in the spinal cord at cervical level, respectively, for all the human models. To better quantify these distributions, Table 3 reports 
the mean and the maximum of the $\mathbf{J}$ current distribution on the spinal cord and/or cauda equina for all the human models and electrode montages. Blank cells in the table mean that the spinal cord or the cauda equina at that level along the spine is not segmented in the respective human models and only cerebrospinal fluid is present at that level along the spine. For all the models, the higher J-field amplitude were generated by Montage $C$ at cervical level. Montage A and Montage B generate at thoracic level comparable field amplitude levels, whereas the Montage $C$ generates field amplitude levels at thoracic level remarkably higher (around the double on average) than the other two montages. At lumbar level, Montage $\mathrm{A}$ and $\mathrm{C}$ generate comparable field levels, but always lower than Montage B.

Interestingly, for all the montages the $\mathbf{J}$ field amplitudes tend to be primarily directed longitudinally along the spinal cord and the cauda equina. This trend is shown in Table 4 that reports the mean of the ratio $(R)$ evaluated slice by slice along the vertebral column between the longitudinal and transversal (i.e. the root square of the quadratic sum of the dorsoventral and mediolateral components) field components at cervical, thoracic, lumbar and sacral/ coccygeal level, for all the human models and electrode montages. Data in the table show that the ratio between the longitudinal and transversal field components varies along the spine and across the human models and electrode montages, but that the longitudinal component is always higher, with the only exception of Montage

Table 4

Mean of the ratio $(R)$ between the longitudinal and the transversal amplitude field components in the spinal cord or cauda equina varying the human models and the electrode montages at different levels along the vertebral column. Blank cells mean that the spinal cord or cauda equine at that level is not segmented.

\begin{tabular}{llll}
\hline & Montage A & Montage B & Montage C \\
\hline Ella & & & \\
Cervical & 0.50 & 0.90 & 6.83 \\
Thoracic & 3.69 & 1.61 & 4.80 \\
Lumbar & & & \\
Sacral \& coccygeal & & & \\
Louis & & & \\
Cervical & 0.30 & 1.27 & 6.98 \\
Thoracic & 1.28 & 1.03 & 1.73 \\
Lumbar & 1.59 & 3.08 & \\
Sacral \& coccygeal & & & 3.51 \\
Billie & & & 4.95 \\
Cervical & 0.49 & 1.24 & 3.16 \\
Thoracic & 1.92 & 1.26 & 2.35 \\
Lumbar & 5.25 & 4.54 & \\
Sacral \& coccygeal & 1.96 & 2.53 & \\
\hline
\end{tabular}

A at cervical level for all the human models and Montage B always at cervical level but only for the "Ella". Among the electrode montages, Montage B tends to induce a stimulation at thoracic level with almost comparable longitudinal and transversal components.

As to the field amplitude distributions on transversal sections of the spinal cord (see Fig. 6, bottom row for Louis as example) and/or cauda equina, Table 5 reports the mean and the maximum of the coefficient of variation (CV) (i.e. the ratio between the standard deviation and the mean) of the $\mathbf{J}$ field amplitude distribution at different levels along the spine for all the models and electrode montages. The field amplitude distribution obtained at the thoracic level is always more uniform on transversal sections (mean CV less than $8 \%$ varying models and electrode montages) than in the other regions, whereas the higher variations were found mainly at cervical levels. Across the electrode montages, Montage B tends to induce a stimulation more homogenous throughout the spinal cord at thoracic level (minimum mean CV across the montages for all the models). This confirm also the rationale of an orthogonal elec-trode montages (with the stimulating electrodes placed epidural on the dorsal spinal cord and referenced to the abdomen) tested on anesthetized rats (Aguilar et al., 2011).

To better investigate what is the relation between the $\mathbf{J}$ distribution on the spinal cord and some tissues close to it, Fig. 7 shows the mean value of $\mathbf{J}$-field amplitude distributions evaluated at different levels along the vertebral column for spinal cord/cauda equina, nerves and muscle of the back along the spine for all the electrode montages and human models. Panels of the figure show that the muscles of the back along the spine are always subjected to mean $\mathbf{J}$ amplitude much more high (see also Fig. 8, that shows some examples of the $\mathbf{J}$ amplitude distributions for Louis shown as sagittal sections across the muscle on the back) than the spinal cord/ cauda equina at the same level along the spine. More interestingly, "Louis", i.e. where the nerves roots are well segmented along the spine, shows that at thoracic level the nerves roots are exposed to mean amplitude values higher (up to 4 times higher for Montage A and $B$ ) than the spinal cord at the same level along the spine. "Billie", indeed, shows that at sacral/coccygeal level only the Montage B can induce in the beginning of the sciatic nerve $\mathbf{J}$ field amplitudes higher than in the cauda equina.

\section{Discussion}

We here reported the first modeling study on the current density generated by transcutaneous spinal DC stimulation in humans. Despite some inter-individual differences due to anatomical vari-

Table 5

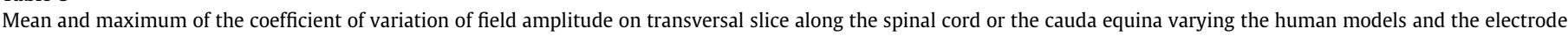
montages. Blank cells mean that the spinal cord or the cauda equina at that level along the spine is not segmented.

\begin{tabular}{|c|c|c|c|c|c|c|}
\hline & \multicolumn{2}{|l|}{ Montage A } & \multicolumn{2}{|c|}{ Montage B } & \multicolumn{2}{|l|}{ Montage C } \\
\hline & Mean (\%) & $\operatorname{Max}(\%)$ & Mean (\%) & $\operatorname{Max}(\%)$ & Mean (\%) & $\operatorname{Max}(\%)$ \\
\hline \multicolumn{7}{|l|}{ Ella } \\
\hline Cervical & 13.5 & 67.2 & 13.6 & 62.7 & 11.1 & 56.6 \\
\hline Thoracic & 7.7 & 29.5 & 7.0 & 33.8 & 7.4 & 29.4 \\
\hline \multicolumn{7}{|l|}{ Lumbar } \\
\hline \multicolumn{7}{|l|}{ Sacral \& coccygeal } \\
\hline \multicolumn{7}{|l|}{ Louis } \\
\hline Cervical & 13.9 & 33.8 & 11.9 & 69.9 & 10.7 & 47.2 \\
\hline Thoracic & 6.1 & 13.3 & 5.5 & 12.7 & 5.5 & 13.1 \\
\hline Lumbar & 8.1 & 16.2 & 6.2 & 13.3 & 8.0 & 18.6 \\
\hline \multicolumn{7}{|l|}{ Sacral \& coccygeal } \\
\hline \multicolumn{7}{|l|}{ Billie } \\
\hline Cervical & 11.8 & 46.0 & 10.3 & 22.0 & 8.2 & 20.8 \\
\hline Thoracic & 7.0 & 18.0 & 5.5 & 14.1 & 6.3 & 14.7 \\
\hline Lumbar & 10.4 & 37.9 & 11.0 & 31.3 & 10.8 & 41.8 \\
\hline Sacral \& coccygeal & 13.4 & 28.6 & 12.8 & 22.2 & 12.0 & 21.4 \\
\hline
\end{tabular}



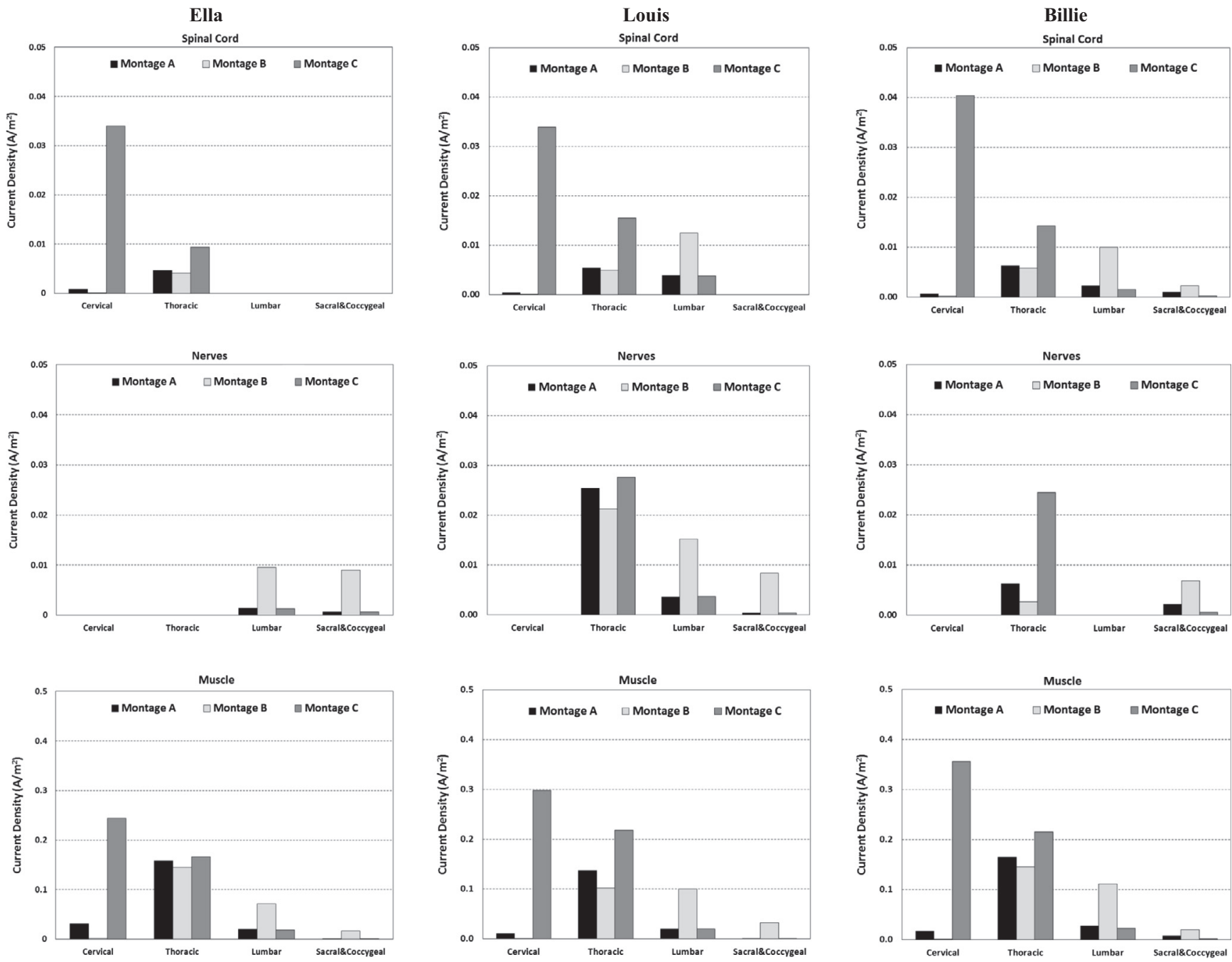

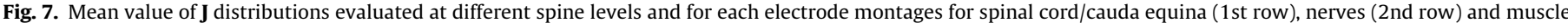

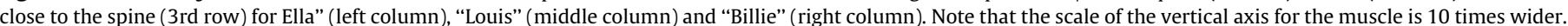

Louis

\begin{tabular}{|c|c|c|c|c|c|}
\hline & Montage A & Montage B & Montage C \\
\hline & & & & & \\
\hline
\end{tabular}

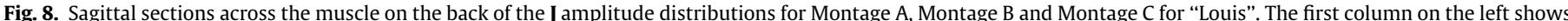

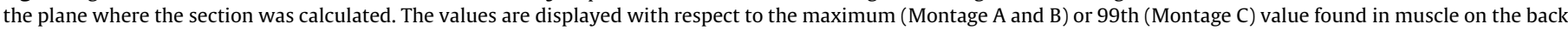
for each electrode montage. 
ability, the region of the spinal cord and of the cauda equina where the current density amplitude distribution is higher, is influenced by the position of the reference electrode, i.e., at thoracic, lumbar or cervical level for a reference electrode on the right arm (Montage A), umbilicus (Montage B) and $\mathrm{Cz}$ (Montage $\mathrm{C}$ ), respec-tively (Figs. 3-6). Interestingly, the J-field distributions found for Montage A are in line with the hypothesis proposed by Cogiamanian et al. (2008) that since this anodal tsDCS montage leaves the median nerve somatosensory-evoked potentials unchanged, it should acts mainly at thoracic level with minimal spread of the current toward the higher spinal cord levels or to the brainstem. On the contrary, the field distributions found for Montage $C$ suggest that it could act also supraspinally.

Within the spinal cord and the cauda equina, tsDCS produces mainly longitudinal $\mathbf{J}$ (and $\mathbf{E}$ ) field amplitudes along almost all the vertebral column, particularly for Montages A and C. This observation could be important in a future possible application of tsDCS on SCI patients. Indeed, available literature (Rajnicek et al., 1998; Hernández-Labrado et al., 2011; Heo et al., 2011) seems to indicate that longitudinal fields of adequate amplitude (of about 5 $\mathrm{mV} / \mathrm{mm}$ ) across the injured spinal cord could have a chance to promote axonal regrowth and to prevent axon degener-ation improving, therefore, the histopathological outcome after SCI (Hernández-Labrado et al., 2011). Rescaling our results (see for example Table 3 ) to electric field amplitude through the inverse of Eq. (3), showed that our E-field amplitudes are on average much lower than the aforementioned threshold, and with the only exception of the maximum amplitudes obtained in the cervical region for the Montage $C$ that resulted close to the threshold. However, it should be noted that the prediction of the peak values can be especially influenced by an intrinsic level of uncertainty due to numerical artifacts, which are introduced, for example, by the staircasing error (Chen et al., 2013).

In all models, our computations show that the field amplitude distributions on transversal sections of the spinal cord at thoracic level were characterized by a mean coefficient of variation less than $8 \%$ (see Table 5), for all the electrode montages. This means that the ventral (motor) and dorsal (sensory) axonal tracts at the same height along the spine experience similar stimulation inten-sity. This results is in line with clinical studies (Cogiamanian et al., 2008, 2012; Winkler et al., 2010; Lamy et al., 2012) showing that tsDCS could affect somatosensory, nociceptive and motor path-ways in the human spinal cord. Our computations, however, could be partially influenced by the small dimension of the spinal struc-ture and by the limited number of voxels that described it.

One shortcoming of this study is that our model of the spinal cord is a simplification of this anatomical structure. Indeed, we represented the spinal cord as a homogenous elongated solid with an almost ellipsoidal transversal section, not distinguishing between the $\mathrm{H}$-shaped central gray matter, the white matter on the periphery of the cord, the meninges and the blood vessels. Since the presence of all these structures has the potential to change current direction and/or intensity, it is not to exclude that better modeling of the spinal cord could partially modify these results.

For all the montages and human models, the levels of current density here found (with a maximum equal to $0.085 \mathrm{~A} / \mathrm{m}^{2}$ at cervical level, see Table 3 ) are still far under the threshold of current density used for invasive spinal cord stimulation $\left(23 \mathrm{~A} / \mathrm{m}^{2}\right)$, (Wesselink et al., 1998) and well below the threshold for neural tissue damage $\left(250 \mathrm{~A} / \mathrm{m}^{2}\right)$ due to pulsed electric stimulation (Cogiamanian et al., 2012; McCreery et al., 1990). Up till now, even if safety data for tsDCS are small, no spinal-specific adverse events have been reported after tsDCS (Cogiamanian et al., 2012). Moreover, direct harmful effects of tsDCS over spinal cord have been excluded by assaying serum neuron specific enolase (NSE) before and immediately after stimulation offset (Cogiamanian et al., 2008).

This study, moreover, shows that due to the distance between the electrode and the target tissue, there is also a dispersion of current in the other tissues surrounding the spinal cord, mainly the muscle on the back and the nerve roots.

\section{Acknowledgement}

The authors wish to thank Schmid \& Partner Engineering AG (www.speag.com) for having provided the simulation software SEMCAD X.

Alberto Priori, Filippo Cogiamanian and Maurizio Vergari are stakeholders of Newronika s.r.l., a spin-off company of the Fondazione IRCCS Ca' Granda Ospedale Maggiore Policlinico and of the Università degli Studi di Milano. All the other authors report no financial interest or potential conflict of interests.

\section{References}

Aguilar J, Pulecchi F, Dilena R, Oliviero A, Priori A, Foffani G. Spinal direct current stimulation modulates the activity of gracile nucleus and primary somatosensory cortex in anaesthetized rats. J Physiol 2011;589:4981-96. Ahmed Z. Trans-spinal direct current stimulation modulates motor cortex-induced muscle contraction in mice. J Appl Physiol 2011;110:1414-24.

Ahmed Z, Wieraszko A. Trans-spinal direct current enhances corticospinal output and stimulation-evoked release of glutamate analog, D-2,3-(3)H-aspartic acid. J Appl Physiol 2012;112:1576-92.

Ahmed Z. Effects of cathodal trans-spinal direct current stimulation on mouse spinal network and complex multijoint movements. J Neurosci 2013a;33:14949-57.

Ahmed Z. Electrophysiological characterization of spino-sciatic and cortico-sciatic associative plasticity: modulation by trans-spinal direct current and effects on recovery after spinal cord injury in mice. J Neurosci 2013b;33:4935-46.

Brunoni AR, Nitsche MA, Bolognini N, Bikson M, Wagner T, Merabet L, et al. Clinical research with transcranial direct current stimulation (tDCS): challenges and future directions. Brain Stimul 2012;5:175-95.

Chen XL, Benkler S, Chavannes N, De Santis V, Bakker J, van Rhoon G, et al. Analysis of human brain exposure to low-frequency magnetic fields: a numerical assessment of spatially averaged electric fields and exposure limits. Bioelectromagnetics 2013;34:375-84.

Cogiamanian F, Vergari M, Pulecchi F, Marceglia S, Priori A. Effect of spinal transcutaneous direct current stimulation on somatosensory evoked potentials in humans. Clin Neurophysiol 2008;119:2636-40.

Cogiamanian F, Vergari M, Schiaffi E, Marceglia S, Ardolino G, Barbieri S, et al. Transcutaneous spinal cord direct current stimulation inhibits the lower limb nociceptive flexion reflex in human beings. Pain 2011;152:370-5.

Cogiamanian F, Ardolino G, Vergari M, Ferrucci R, Ciocca M, Scelzo E, et al. Transcutaneous spinal direct current stimulation. Front Psychiatry 2012;3:63.

Christ A, Kainz W, Hahn EG, Honegger K, Zefferer M, Neufeld E, et al. The virtual family-development of surface-based anatomical models of two adults and two

children for dosimetric simulations. Phys Med Biol 2010;55:N23-38. Dimbylow P.

Development of the female voxel phantom, NAOMI, and its application to calculations of induced current densities and electric fields from applied low frequency magnetic and electric fields. Phys Med Biol 2005;50:1047-70.

Gabriel C, Peyman A, Grant EH. Electrical conductivity of tissue at frequencies below $1 \mathrm{MHz}$. Phys Med Biol 2009;54:4863-78.

Gabriel C. Comments on dielectric properties of the skin. Phys Med Biol 1997;42:1671-4.

Gabriel S, Lau RW, Gabriel C. The dielectric properties of biological tissues: II. Measurements in the frequency range $10 \mathrm{~Hz}$ to $20 \mathrm{GHz}$. Phys Med Biol 1996;41:2251-69.

ICNIRP guidelines for limiting exposure to time-varying electric and magnetic fields ( $1 \mathrm{~Hz}$ to $100 \mathrm{kHz}$ ). Health Phys 2010;99:818-36.

Hernández-Labrado GR, Polo JL, López-Dolado E, Collazos-Castro JE. Spinal cord direct current stimulation: finite element analysis of the electric field and current density. Med Biol Eng Comput 2011;49:417-29.

Heo C, Yoo J, Lee S, Jo A, Jung S, Yoo $\mathrm{H}$, et al. The control of neural cell-to-cell interactions through noncontact electrical field stimulation using graphene electrodes. Biomaterials 2011;32:19-27.

Hubli M, Dietz V, Schrafl-Altermatt M, Bolliger M. Modulation of spinal neuronal excitability by spinal direct currents and locomotion after spinal cord injury. Clin Neurophysiol 2013;124:1187-95.

Lamy JC. Seeking significance for transcutaneous spinal DC stimulation. Clin Neurophysiol 2013;124:1049-50.

Lamy JC, Ho C, Badel A, Arrigo RT, Boakye M. Modulation of Soleus H-reflex by spinal DC stimulation in humans. J Neurophysiol 2012;108:906-14. 
McCreery DB, Agnew WF, Yuen TG, Bullara L. Charge density and charge per phase as cofactors in neural injury induced by electrical stimulation. IEEE Trans Biomed Eng 1990;37:996-1001.

Parazzini M, Fiocchi S, Rossi E, Paglialonga A, Ravazzani P. Transcranial direct current stimulation: estimation of the electric field and of the current density in an anatomical human head model. IEEE Trans Biomed Eng 2011;58:1773-80.

Parazzini M, Fiocchi S, Ravazzani P. Electric field and current density distribution in an anatomical head model during transcranial direct current stimulation for tinnitus treatment. Bioelectromagnetics 2012;33:476-87.

Rajnicek AM, Robinson KR, McCaig CD. The direction of neurite growth in a weak DC electric field depends on the substratum: contributions of adhesivity and net surface charge. Dev Biol 1998;203:412-23.
Truini A, Vergari M, Biasiotta A, La Cesa S, Gabriele M, Di Stefano G, et al. Transcutaneous spinal direct current stimulation inhibits nociceptive spinal pathway conduction and increases pain tolerance in humans. Eur J Pain 2011;15:1023-7.

Vergari M, Cogiamanian F, Di Bisceglie M, Cognetto V, Ardolino G, Ciocca M, et al. Additive after effects of spinal and cortical DC stimulation on human flexion

reflex pathways. J Peripher Nerv Syst 2012;17. S57-S57.

Wesselink WA, Holsheimer J, Boom HBK. Analysis of current density and related parameters in spinal cord stimulation. IEEE Trans Rehabil Eng 1998;6:200-7.

Winkler T, Hering P, Straube A. Spinal DC stimulation in humans modulates postactivation depression of the H-reflex depending on current polarity. Clin Neurophysiol 2010;121:957-61. 